\title{
EXTENSION OF THE METHOD OF QUASILINEARIZATION FOR STOCHASTIC INITIAL VALUE PROBLEMS
}

\author{
N. SHAHZAD ${ }^{1}$ \\ Florida Institute of Technology \\ Department of Applied Mathematics \\ Melbourne, Florida 32901 USA \\ FARZANA A. MCRAE \\ Jacksonville University \\ Department of Mathematics \\ Jacksonville, Florida 32211 USA
}

(Received August, 1994; revised November, 1994)

\begin{abstract}
In this paper we extend the method of quasilinearization to stochastic initial value problems. Further we prove that the iterates converge uniformly almost surely to the unique solution and the convergence is quadratic.
\end{abstract}

Key words: Quasilinearization, quadratic convergence, monotone sequence, stochastic initial value problem.

AMS (MOS) subject classifications: 60H25, 34A34

\section{Introduction}

Quasilinearization is a well known technique for obtaining approximate solutions of nonlinear differential equations [1, 2]. It provides a monotone sequence of approximate solutions that converges quadratically to the unique solution of the IVP (initial value problem)

$$
u^{\prime}=f(t, u), u(0)=u_{0} \text { on } J=[0, T]
$$

if $f$ is convex. Recently, this method has been generalized and extended using less restrictive conditions on $f$ so as to be applicable to a large class of problems [4-10, 12]. In particular, in [4, 8], this technique has been extended to obtain monotone sequences that converge quadratically to the unique solution of (1.1) when $f$ can be decomposed into a difference of two convex functions. In this paper we extend the technique used in [8] to stochastic initial value problems.

\section{Main Result}

Let $(\Omega, \mathcal{A}, P)$ be a probability measure space and $u_{0}: \Omega \longrightarrow \mathbb{R}$ be a given measurable function. Consider the stochastic initial value problem (SIVP)

\footnotetext{
${ }^{1}$ On leave from the Department of Mathematics, Quaid-i-Azam University, Islamabad, Pakistan.
} 


$$
\begin{gathered}
u^{\prime}(t, \omega)=f(t, u(t, \omega), \omega)+g(t, u(t, \omega), \omega), \text { a.e. on } J=[0, T] \\
u(0, \omega)=u_{0}(\omega),
\end{gathered}
$$

where $f: J \times \mathbb{R} \times \Omega \longrightarrow \mathbb{R}$ and $g: J \times \mathbb{R} \times \Omega \longrightarrow \mathbb{R}$ satisfy:

(i) $\quad f(t, u, \cdot)$ and $g(t, u, \cdot)$ are measurable for all $(t, u)$;

(ii) $\quad f(\cdot, u, \cdot)$ and $g(\cdot, u, \cdot)$ are measurable for every $u$;

(iii) $f(t, \cdot, \omega)$ and $g(t, \cdot, \omega)$ are continuous for all $(t, \omega)$.

Suppose that

1) $|f(t, x, \omega)| \leq K(t, \omega)$ on $J \times \mathbb{R} \times \Omega$, where $K: J \times \Omega \longrightarrow \mathbb{R}_{+}$is measurable in $t$ and $\int_{0}^{T} K(s, \omega) d s<\infty$ on $\Omega$.

A stochastic process $u: J \times \Omega \longrightarrow \mathbb{R}$ is called a sample solution of $(2.1)$ if $u(0, \cdot)=u_{0}$ and is absolutely continuous (a.c.) on $J$ and satisfies $u^{\prime}(t, \omega)=f(t, u(t, \omega), \omega)+g(t, u(t, \omega), \omega)$, a.e. on $J$.

A stochastic process $\alpha: J \times \Omega \longrightarrow \mathbb{R}$ is said to be a sample lower solution of (2.1) if for almost all $\omega \in \Omega, \alpha(\cdot, \omega)$ is a.c. and $\alpha^{\prime}(t, \omega) \leq f(t, \alpha(t, \omega), \omega)+g(t, \alpha(t, \omega), \omega)$, a.e. on $J$. The definition of sample upper solution is obtained by reversing the inequality above. For further details we refer to $[3]$.

Theorem 2.1: Assume that

$\left.A_{1}\right) \quad \alpha_{0}$ and $\beta_{0}$ are lower and upper sample solutions of (2.1) such that $\alpha_{0} \leq \beta_{0}$ on $J \times \Omega$;

in

$\left.A_{2}\right) \quad f_{u}(t, u, \omega), g_{u}(t, u, \omega), f_{u u}(t, u, \omega), g_{u u}(t, u, \omega)$ exist, are continuous in $u$, measurable

$$
\omega \text {, measurable in }(t, \omega) \text { and satisfy } f_{u u}(t, u, \omega) \geq 0, g_{u u}(t, u, \omega) \leq 0 \text {; }
$$

$\left.A_{3}\right) \quad f_{u}, g_{u}, f_{u u}$ and $g_{u u}$ satisfy (2.1) with different bounds.

Then there exist monotone sequences $\left\{\alpha_{n}(t, \omega)\right\},\left\{\beta_{n}(t, \omega)\right\}$ which converge uniformly, for almost all $\omega \in \Omega$, to the unique sample solution of (2.1) and the convergence is quadratic.

Proof: Let us first observe that $\left(A_{2}\right)$ implies, for any $u \geq v$,

$$
\left\{\begin{aligned}
f(t, u, \omega) & \geq f(t, v, \omega)+f_{u}(t, v, \omega)(u-v) \\
g(t, u, \omega) & \geq g(t, v, \omega)+g_{u}(t, u, \omega)(u-v)
\end{aligned}\right.
$$

Moreover, for any $u_{1}, u_{2}$ such that $\alpha_{0}(t, \omega) \leq u_{2} \leq u_{1} \leq \beta_{0}(t, \omega)$, it follows that

$$
\left\{\begin{array}{r}
f\left(t, u_{1}, \omega\right)-f\left(t, u_{2}, \omega\right) \leq L_{1}(t, \omega)\left(u_{1}-u_{2}\right) \\
g\left(t, u_{1}, \omega\right)-g\left(t, u_{2}, \omega\right) \leq L_{2}(t, \omega)\left(u_{1}-u_{2}\right)
\end{array}\right.
$$

a.e. on $J$, where $L_{i}(t, \omega)>0$, is measurable for every $t$ and $\int_{0}^{T} L_{i}(t, \omega) d s<\infty$ on $\Omega$, for $i=1,2$.

Let $\alpha_{1}(t, \omega), \beta_{1}(t, \omega)$ be sample solutions of the linear SIVPs

$$
\left\{\begin{array}{l}
\alpha_{1}^{\prime}=f\left(t, \alpha_{0}, \omega\right)+f_{u}\left(t, \alpha_{0}, \omega\right)\left(\alpha_{1}-\alpha_{0}\right)+g\left(t, \alpha_{0}, \omega\right)+g_{u}\left(t, \beta_{0}, \omega\right)\left(\alpha_{1}-\alpha_{0}\right), \alpha_{1}(0, \omega)=u_{0}(\omega) \\
\beta_{1}^{\prime}=f\left(t, \beta_{0}, \omega\right)+f_{u}\left(t, \alpha_{0}, \omega\right)\left(\beta_{1}-\beta_{0}\right)+g\left(t, \beta_{0}, \omega\right)+g_{u}\left(t, \beta_{0}, \omega\right)\left(\beta_{1}-\beta_{0}\right), \beta_{1}(0, \omega)=u_{0}(\omega)
\end{array}\right.
$$

a.e. on $J$, where $\alpha_{0}(0, \omega) \leq u_{0}(\omega) \leq \beta_{0}(0, \omega)$.

We shall prove that $\alpha_{0} \leq \alpha_{1}$ on $J \times \Omega$. To do this, let $p=\alpha_{0}-\alpha_{1}$ so that $p(0, \omega) \leq 0$. Then, 
using (2.4), we get

$$
\begin{aligned}
p^{\prime}= & \alpha_{0}^{\prime}-\alpha_{1}^{\prime} \\
\leq & f\left(t, \alpha_{0}, \omega\right)+g\left(t, \alpha_{0}, \omega\right)-\left[f\left(t, \alpha_{0}, \omega\right)+f_{u}\left(t, \alpha_{0}, \omega\right)\left(\alpha_{1}-\alpha_{0}\right)\right. \\
& \left.+g\left(t, \alpha_{0}, \omega\right)+g_{u}\left(t, \beta_{0}, \omega\right)\left(\alpha_{1}-\alpha_{0}\right)\right] \\
= & {\left[f_{u}\left(t, \alpha_{0}, \omega\right)+g_{u}\left(t, \beta_{0}, \omega\right)\right] p, \text { a.e. on } J . }
\end{aligned}
$$

This implies, by Theorem $1.1[11]$, that $p(t, \omega) \leq 0$ on $J \times \Omega$. Now set $p=\alpha_{1}-\beta_{0}$ and note that $p(0, \omega) \leq 0$. Using $(2.2)$ and $(2.4)$, we obtain

$$
\begin{aligned}
p^{\prime}= & \alpha_{1}^{\prime}-\beta_{0}^{\prime} \\
\leq & {\left[f\left(t, \alpha_{0}, \omega\right)+f_{u}\left(t, \alpha_{0}, \omega\right)\left(\alpha_{1}-\alpha_{0}\right)+g\left(t, \alpha_{0}, \omega\right)+g_{u}\left(t, \beta_{0}, \omega\right)\left(\alpha_{1}-\alpha_{0}\right)\right] } \\
& -f\left(t, \beta_{0}, \omega\right)-g\left(t, \beta_{0}, \omega\right) \\
\leq & {\left[f\left(t, \alpha_{0}, \omega\right)+f_{u}\left(t, \alpha_{0}, \omega\right)\left(\alpha_{1}-\alpha_{0}\right)+g\left(t, \beta_{0}, \omega\right)-g_{u}\left(t, \beta_{0}, \omega\right)\left(\beta_{0}-\alpha_{0}\right)\right.} \\
& \left.+g_{u}\left(t, \beta_{0}, \omega\right)\left(\alpha_{1}-\alpha_{0}\right)\right]-f\left(t, \alpha_{0}, \omega\right)-f_{u}\left(t, \alpha_{0}, \omega\right)\left(\beta_{0}-\alpha_{0}\right)-g\left(t, \beta_{0}, \omega\right) \\
= & {\left[f_{u}\left(t, \alpha_{0}, \omega\right)+g_{u}\left(t, \beta_{0}, \omega\right)\right] p, \text { a.e. on } J, }
\end{aligned}
$$

which again implies $p(t, \omega) \leq 0$ on $J \times \Omega$. As a result we have $\alpha_{0}(t, \omega) \leq \alpha_{1}(t, \omega) \leq \beta_{0}(t, \omega)$ on $J \times \Omega$. Similarly, we can find that $\alpha_{0}(t, \omega) \leq \beta_{1}(t, \omega) \leq \beta_{0}(t, \omega)$ on $J \times \Omega$. We need to show that $\alpha_{1}(t, \omega) \leq \beta_{11}(t, \omega)$ on $J \times \Omega$ so that it yields

$$
\alpha_{0}(t, \omega) \leq \alpha_{1}(t, \omega) \leq \beta_{1}(t, \omega) \leq \beta_{0}(t, \omega) \text { on } J \times \Omega \text {. }
$$

Using (2.2) and (2.4), we see that

$$
\begin{gathered}
\alpha_{1}^{\prime}=f\left(t, \alpha_{0}, \omega\right)+f_{u}\left(t, \alpha_{0}, \omega\right)\left(\alpha_{1}-\alpha_{0}\right)+g\left(t, \alpha_{0}, \omega\right)+g_{u}\left(t, \beta_{0}, \omega\right)\left(\alpha_{1}-\alpha_{0}\right) \\
\leq f\left(t, \alpha_{1}, \omega\right)+g\left(t, \alpha_{1}, \omega\right)-g_{u}\left(t, \alpha_{1}, \omega\right)\left(\alpha_{1}-\alpha_{0}\right)+g_{u}\left(t, \beta_{0}, \omega\right)\left(\alpha_{1}-\alpha_{0}\right) \\
=f\left(t, \alpha_{1}, \omega\right)+g\left(t, \alpha_{1}, \omega\right)+\left[g_{u}\left(t, \beta_{0}, \omega\right)-g_{u}\left(t, \alpha_{1}, \omega\right)\right]\left(\alpha_{1}-\alpha_{0}\right) \\
\leq f\left(t, \alpha_{1}, \omega\right)+g\left(t, \alpha_{1}, \omega\right) \text {, a.e. on } J,
\end{gathered}
$$

because of the fact that $g_{u}(t, u, \omega)$ is nonincreasing in $u$ and $\alpha_{1} \leq \beta_{0}$ on $J \times \Omega$. Similarly, using (2.2) again, we obtain

$$
\begin{gathered}
\beta_{1}^{\prime}=f\left(t, \beta_{0}, \omega\right)+f_{u}\left(t, \alpha_{0}, \omega\right)\left(\beta_{1}-\beta_{0}\right)+g\left(t, \beta_{0}, \omega\right)+g_{u}\left(t, \beta_{0}, \omega\right)\left(\beta_{1}-\beta_{0}\right) \\
\geq f\left(t, \beta_{1}, \omega\right)+f_{u}\left(t, \beta_{1}, \omega\right)\left(\beta_{1}-\beta_{0}\right)+f_{u}\left(t, \alpha_{0}, \omega\right)\left(\beta_{1}-\beta_{0}\right)+g\left(t, \beta_{1}, \omega\right) \\
=f\left(t, \beta_{1}, \omega\right)+\left[-f_{u}\left(t, \beta_{1}, \omega\right)+f_{u}\left(t, \alpha_{0}, \omega\right)\right]\left(\beta_{1}-\beta_{0}\right)+g\left(t, \beta_{1}, \omega\right) \\
\geq f\left(t, \beta_{1}, \omega\right)+g\left(t, \beta_{1}, \omega\right), \text { a.e. on } J
\end{gathered}
$$

because of the fact that $f_{u}(t, u, \omega)$ is nondecreasing in $u$ and $\alpha_{0} \leq \beta_{1}$ on $J \times \Omega$. It then follows 
from Theorem 1.1 [11] and (2.3), that $\alpha_{1}(t, \omega) \leq \beta_{1}(t, \omega)$ on $J \times \Omega$ which shows that (2.5) is valid.

Assume that for some $k>1, \alpha_{k}^{\prime} \leq f\left(t, \alpha_{k}, \omega\right)+g\left(t, \alpha_{k}, \omega\right), \beta_{k}^{\prime} \geq f\left(t, \beta_{k}, \omega\right)+g\left(t, \beta_{k}, \omega\right)$, a.e. on $J$ and $\alpha_{k}(t, \omega) \leq \beta_{k}(t, \omega)$ on $J \times \Omega$. We shall prove that

$$
\alpha_{k}(t, \omega) \leq \alpha_{k+1}(t, \omega) \leq \beta_{k+1}(t, \omega) \leq \beta_{k}(t, \omega) \text { on } J \times \Omega
$$

where $\alpha_{k+1}(t, \omega)$ and $\beta_{k+1}(t, \omega)$ are sample solutions of the linear stochastic SIVPs

$$
\begin{aligned}
& \alpha_{k+1}^{\prime}=f\left(t, \alpha_{k}, \omega\right)+f_{u}\left(t, \alpha_{k}, \omega\right)\left(\alpha_{k+1}-\alpha_{k}\right)+g\left(t, \alpha_{k}, \omega\right) \\
& +g_{u}\left(t, \beta_{k}, \omega\right)\left(\alpha_{k+1}-\alpha_{k}\right), \\
& \alpha_{k+1}(0, \omega)=u_{0}(\omega)
\end{aligned}
$$

and

$$
\begin{aligned}
& \beta_{k+1}^{\prime}=f\left(t, \beta_{k}, \omega\right)+f_{u}\left(t, \alpha_{k}, \omega\right)\left(\beta_{k+1}-\beta_{k}\right)+g\left(t, \beta_{k}, \omega\right) \\
& \quad+g_{u}\left(t, \beta_{k}, \omega\right)\left(\beta_{k+1}-\beta_{k}\right), \\
& \beta_{k+1}(0, \omega)=u_{0}(\omega),
\end{aligned}
$$

a.e. on $J$.

Setting $p=\alpha_{k}-\alpha_{k+1}$, we have, as before, that $p^{\prime} \leq\left[f_{u}\left(t, \alpha_{k}, \omega\right)+g_{u}\left(t, \beta_{k}, \omega\right)\right] p$, a.e. on $J$ and $p(0, \omega)=0$. This proves that $p(t, \omega) \leq 0$ on $J \times \Omega$. On the other hand, letting $p=\alpha_{k+1}-\beta_{k}$ yields

$$
\begin{aligned}
p^{\prime}= & \alpha_{k+1}^{\prime}-\beta_{k}^{\prime} \\
\leq & f\left(t, \alpha_{k}, \omega\right)+f_{u}\left(t, \alpha_{k}, \omega\right)\left(\alpha_{k+1}-\alpha_{k}\right)+g\left(t, \alpha_{k}, \omega\right)+g_{u}\left(t, \beta_{k}, \omega\right)\left(\alpha_{k+1}-\alpha_{k}\right) \\
& \quad-f\left(t, \beta_{k}, \omega\right)-g\left(t, \beta_{k}, \omega\right) .
\end{aligned}
$$

Since $\alpha_{k} \leq \beta_{k},(2.2)$ gives, after some computation,

$$
p^{\prime} \leq\left[f_{u}\left(t, \alpha_{k}, \omega\right)+g_{u}\left(t, \beta_{k}, \omega\right)\right] p, \text { a.e. on } J .
$$

Thus we have $\alpha_{k}(t, \omega) \leq \alpha_{k+1}(t, \omega) \leq \beta_{k}(t, \omega)$ on $J \times \Omega$. Similar arguments yield $\alpha_{k}(t, \omega) \leq$ $\beta_{k+1}(t, \omega) \leq \beta_{k}(t, \omega)$ on $J \times \Omega$. Now to show that $\alpha_{k+1}^{\prime} \leq f\left(t, \alpha_{k+1}, \omega\right)+g\left(t, \alpha_{k+1}, \omega\right)$, we proceed as before. Utilizing $(2.2),(2.7)$ and $\left(A_{2}\right)$, we get

$$
\begin{gathered}
\alpha_{k+1}^{\prime} \leq f\left(t, \alpha_{k+1}, \omega\right)+g\left(t, \alpha_{k+1}, \omega\right)-g_{u}\left(t, \alpha_{k+1}, \omega\right)\left(\alpha_{k+1}-\alpha_{k}\right)+g_{u}\left(t, \beta_{k}, \omega\right)\left(\alpha_{k+1}-\alpha_{k}\right) \\
=f\left(t, \alpha_{k+1}, \omega\right)+g\left(t, \alpha_{k+1}, \omega\right)+\left[g_{u}\left(t, \beta_{k}, \omega\right)-g_{u}\left(t, \alpha_{k+1}, \omega\right)\right]\left(\alpha_{k+1}-\alpha_{k}\right) \\
\leq f\left(t, \alpha_{k+1}, \omega\right)+g\left(t, \alpha_{k+1}, \omega\right), \text { a.e. on } J .
\end{gathered}
$$

In a similar manner, we can prove that $\beta_{k+1}^{\prime} \geq f\left(t, \beta_{k+1}, \omega\right)+g\left(t, \beta_{k+1}, \omega\right)$, a.e. on $J$ and hence Theorem 1.1 [11] shows that $\alpha_{k+1}(t, \omega) \leq \beta_{k+1}(t, \omega)$ on $J \times \Omega$ which proves (2.6) is true. Hence by induction we have for all $n$,

$$
\alpha_{0} \leq \alpha_{1} \leq \alpha_{2} \leq \ldots \leq \alpha_{n} \leq \beta_{n} \leq \ldots \leq \beta_{2} \leq \beta_{1} \leq \beta_{0} \text { on } J \times \Omega .
$$

Let us note that for each fixed $t \in J$, 


$$
\begin{aligned}
& a(t, \omega)=\sup _{n \geq 0} \alpha_{n}(t, \omega)=\lim _{n \rightarrow \infty} \alpha_{n}(t, \omega), \\
& b(t, \omega)=\inf _{n \geq 0} \beta_{n}(t, \omega)=\lim _{n \rightarrow \infty} \beta_{n}(t, \omega)
\end{aligned}
$$

exist and $a(t, \omega), b(t, \omega)$ are measurable functions in $\omega$ for each $t \in J$. We obtain, from (2.7) and $(2.8)$,

$$
\begin{aligned}
& \alpha_{n+1}(t, \omega)=u_{0}(\omega)+\int_{0}^{t}\left[f\left(s, \alpha_{k}(s, \omega), \omega\right)+f_{u}\left(s, \alpha_{k}(s, \omega), \omega\right)\left(\alpha_{k+1}(s, \omega)-\alpha_{k}(s, \omega)\right)\right. \\
& \left.\quad+g\left(s, \alpha_{k}(s, \omega), \omega\right)+g_{u}\left(s, \beta_{k}(s, \omega), \omega\right)\left(\alpha_{k+1}(s, \omega)-\alpha_{k}(s, \omega)\right)\right] d s
\end{aligned}
$$

and

$$
\begin{aligned}
& \beta_{k+1}(t, \omega)=u_{0}(\omega)+\int_{0}^{t}\left[f\left(s, \beta_{k}(s, \omega), \omega\right)+f_{u}\left(s, \alpha_{k}(s, \omega), \omega\right)\left(\beta_{k+1}(s, \omega)-\beta_{k}(s, \omega)\right)\right. \\
& \left.\quad+g\left(\mathrm{~s}, \beta_{k}(s, \omega), \omega\right)+g_{u}\left(s, \beta_{k}(s, \omega), \omega\right)\left(\beta_{k+1}(s, \omega)-\beta_{k}(s, \omega)\right)\right] d s .
\end{aligned}
$$

By standard arguments, it is easily seen that $\left\{\alpha_{n+1}(t, \omega)\right\}$ and $\left\{\beta_{n+1}(t, \omega)\right\}$ are sample bounded and equicontinuous and consequently, (2.3) together with Lebesgue dominated convergence theorem yields that

$$
a(t, \omega)=u_{0}(\omega)+\int_{0}^{t}\{f(s, a(s, \omega), \omega)+g(s, a(s, \omega), \omega)\} d s
$$

and

$$
b(t, \omega)=u_{0}(\omega)+\int_{0}^{t}\{f(s, b(s, \omega), \omega)+g(s, b(s, \omega), \omega)\} d s
$$

In view of (2.3), it is clear that $a=b$ on $J \times \Omega$, and as a result, $a=b=u$ on $J \times \Omega$ is the unique sample solution of $(2.1)$.

Next we shall show that the convergence of the sequences $\left\{\alpha_{n}(t, \omega)\right\},\left\{\beta_{n}(t, \omega)\right\}$ to $u(t, \omega)$ is quadratic. Let $p_{n}(t, \omega)=u(t, \omega)-\alpha_{n}(t, \omega) \geq 0, q_{n}(t, \omega)=\beta_{n}(t, \omega)-u(t, \omega) \geq 0$, and note that $p_{n}(0, \omega)=0, q_{n}(0, \omega)=0$. From $(2.7)$ and the mean value theorem together with $\left(A_{2}\right)$, we obtain successively,

$$
\begin{aligned}
& p_{n}^{\prime}=f(t, u, \omega)+g(t, u, \omega)-\left[f\left(t, \alpha_{n-1}, \omega\right)+f_{u}\left(t, \alpha_{n-1}, \omega\right)\left(\alpha_{n}-\alpha_{n-1}\right)\right. \\
& \left.\quad+g\left(t, \alpha_{n-1}, \omega\right)+g_{u}\left(t, \beta_{n-1}, \omega\right)\left(\alpha_{n}-\alpha_{n-1}\right)\right] \\
& =f_{u}(t, \delta, \omega) p_{n-1}+g_{u}(t, \sigma, \omega) p_{n-1}+f_{u}\left(t, \alpha_{n-1}, \omega\right)\left(-p_{n-1}+p_{n}\right) \\
& \quad+g_{u}\left(t, \beta_{n-1}, \omega\right)\left(-p_{n-1}+p_{n}\right) \\
& \leq\left[f_{u}(t, u, \omega)-f_{u}\left(t, \alpha_{n-1}, \omega\right)\right] p_{n-1}-\left[g_{u}\left(t, \beta_{n-1}, \omega\right)-g_{u}\left(t, \alpha_{n-1}, \omega\right)\right] p_{n-1} \\
& \quad+\left[f_{u}\left(t, \alpha_{n-1}, \omega\right)+g_{u}\left(t, \beta_{n-1}, \omega\right)\right] p_{n} \\
& =f_{u u}\left(t, \delta_{1}, \omega\right) p_{n-1}^{2}-g_{u u}\left(t, \sigma_{1}, \omega\right)\left(\beta_{n-1}-\alpha_{n-1}\right) p_{n-1} \\
& \quad+\left[f_{u}\left(t, \alpha_{n-1}, \omega\right)+g_{u}\left(t, \beta_{n-1}, \omega\right)\right] p_{n},
\end{aligned}
$$


where $\alpha_{n-1}<\delta, \sigma<u$ and $\alpha_{n-1}<\delta_{1}<u, \alpha_{n-1}<\sigma_{1}<\beta_{n-1}$.

But

$$
\begin{gathered}
-g_{u u}\left(t, \sigma_{1}, \omega\right)\left[\beta_{n-1}-\alpha_{n-1}\right] p_{n-1} \leq N_{2}(t, \omega)\left[q_{n-1}+p_{n-1}\right] p_{n-1} \\
=N_{2}(t, \omega)\left[p_{n-1}^{2}+p_{n-1} q_{n-1}\right] \\
\leq 2 N_{2}(t, \omega) p_{n-1}^{2}+N_{2}(t, \omega) q_{n-1}^{2} .
\end{gathered}
$$

Thus

$$
p_{n}^{\prime} \leq M(t, \omega) p_{n}+\left[N_{1}(t, \omega)+2 N_{2}(t, \omega)\right] p_{n-1}^{2}+N_{2}(t, \omega) q_{n-1}^{2},
$$

where $\left|f_{u}(t, u, \omega)\right| \leq M_{1}(t, \omega), \quad\left|g_{u}(t, u, \omega)\right| \leq M_{2}(t, \omega), \quad\left|f_{u u}(t, u, \omega)\right| \leq N_{1}(t, \omega), \quad\left|g_{u u}(t, u, \omega)\right| \leq$ $N_{2}(t, \omega)$,

$$
M=M_{1}+M_{2}, \int_{0}^{T} M(t, \omega) d t=Q(\omega)<\infty, \int_{0}^{T} N_{1}(t, \omega) d t=R(\omega)<\infty
$$

and

$$
\int_{0}^{T} N_{2}(t, \omega) d t=S(\omega)<\infty
$$

Thus, by Gronwall's lemma, we get

$$
\begin{aligned}
0 & \leq p_{n}(t, \omega) \\
& \leq \int_{0}^{t}\left\{\exp \int_{s}^{t} M(s, \omega) d s\right\}\left[\left\{N_{1}(s, \omega)+2 N_{2}(s, \omega)\right\} p_{n-1}^{2}(s, \omega)+N_{2}(s, \omega) q_{n-1}^{2}(s, \omega)\right] d s \\
& \leq \int_{0}^{T}\left\{\exp \int_{0}^{T} M(s, \omega) d s\right\}\left[\left\{N_{1}(s, \omega)+2 N_{2}(s, \omega)\right\} p_{n-1}^{2}(s, \omega)+N_{2}(s, \omega) q_{n-1}^{2}(s, \omega)\right] d s .
\end{aligned}
$$

It therefore follows that

$$
\begin{aligned}
\max _{J}\left|u(t, \omega)-\alpha_{n}(t, \omega)\right| \leq & e^{Q(\omega)}\left[\{R(\omega)+2 S(\omega)\} \max _{J}\left|u(t, \omega)-\alpha_{n-1}(t, \omega)\right|^{2}\right. \\
& \left.+S(\omega) \max _{J}\left|\beta_{n-1}(t, \omega)-u(t, \omega)\right|^{2}\right]
\end{aligned}
$$

for almost all $\omega \in \Omega$.

Similarly we can proved that

$$
\begin{aligned}
\max _{J}\left|\beta_{n}(t, \omega)-u(t, \omega)\right| \leq & e^{Q(\omega)}\left[\{S(\omega)+2 R(\omega)\} \max _{J}\left|u(t, \omega)-\beta_{n-1}(t, \omega)\right|^{2}\right. \\
& \left.+R(\omega) \max _{J}\left|\alpha_{n-1}(t, \omega)-u(t, \omega)\right|^{2}\right]
\end{aligned}
$$

for almost all $\omega \in \Omega$. This completes the proof. 


\section{References}

[1] Bellman, R., Methods of Nonlinear Analysis, Vol. II, Academic Press, New York 1973.

[2] Bellman, R. and Kalaba, R., Quasilinearization and Boundary Value Problems, American Elsevier, New York 1965.

[3] Ladde, G.S. and Lakshmikantham, V., Random Differential Inequalities, Academic Press 1980 .

[4] Lakshmikantham, V., An extension of the method of quasilinearization, (to appear in $J$. Optim. Theory and Appl.).

[5] Lakshmikantham, V., Further improvement of generalized quasilinearization method, (to appear in Nonlinear Analysis).

[6] Lakshmikantham, V. and Köksal, S., Another extension of the method of quasilinearization (to appear in Proceedings of Dynamic Systems and Applications).

[7] Lakshmikantham, V., Leela, S. and McRae, F.A., Improved generalized quasilinearization, (to appear in Nonlinear Analysis).

[8] Lakshmikantham, V., Leela, S. and Sivasundaram, S., Extensions of the method of quasilinearization, (to appear in J. Optim. Theory and Appl.).

[9] Lakshmikantham, V. and Malek, S., Generalized quasilinearization, Nonlinear World 1 (1994), 59-63.

[10] Lakshmikantham, V. and Shahzad, N., Further generalization of generalized quasilinearization method, J. Appl. Math. Stoch. Anal. 7 (1994).

[11] Lakshmikantham, V. and Zhang, B.G., On the method of sample upper and lower solutions for stochastic differential equation, Stoch. Anal. and Appl. 3 (1985), 341-347.

[12] McRae, F.A., Generalized quasilinearization of stochastic initial value problem, Stoch. Anal. and Appl. 13 (1994). 


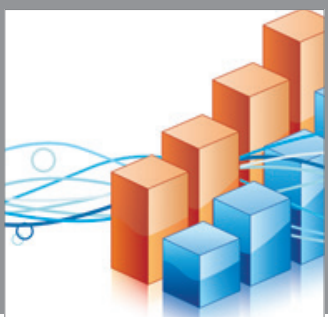

Advances in

Operations Research

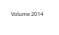

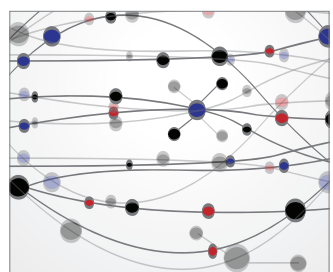

\section{The Scientific} World Journal
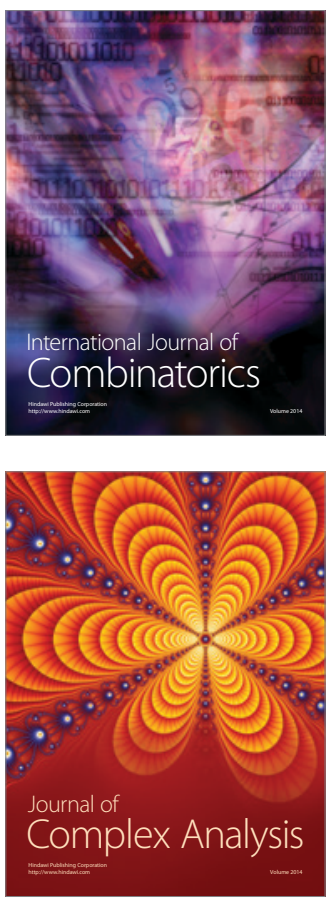

International Journal of

Mathematics and

Mathematical

Sciences
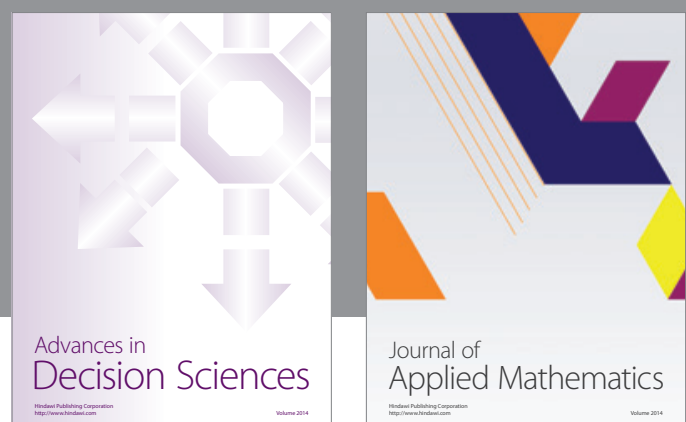

Journal of

Applied Mathematics
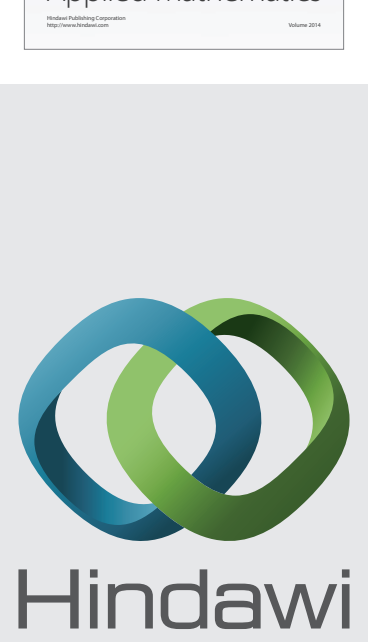

Submit your manuscripts at http://www.hindawi.com
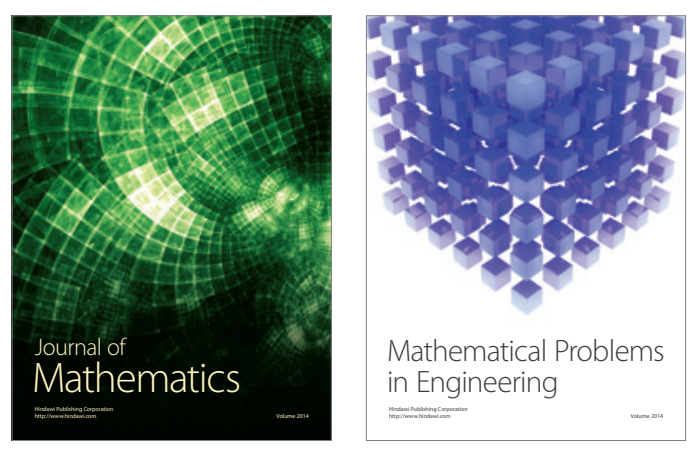

Mathematical Problems in Engineering
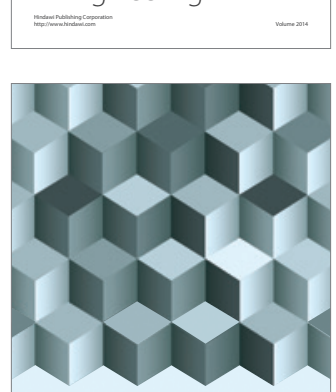

Journal of

Function Spaces
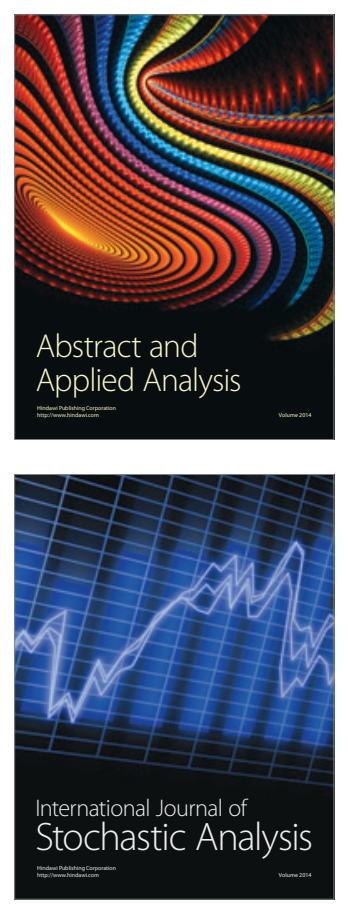

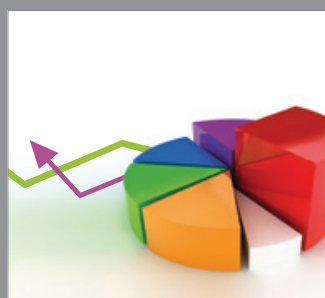

ournal of

Probability and Statistics

Promensencen
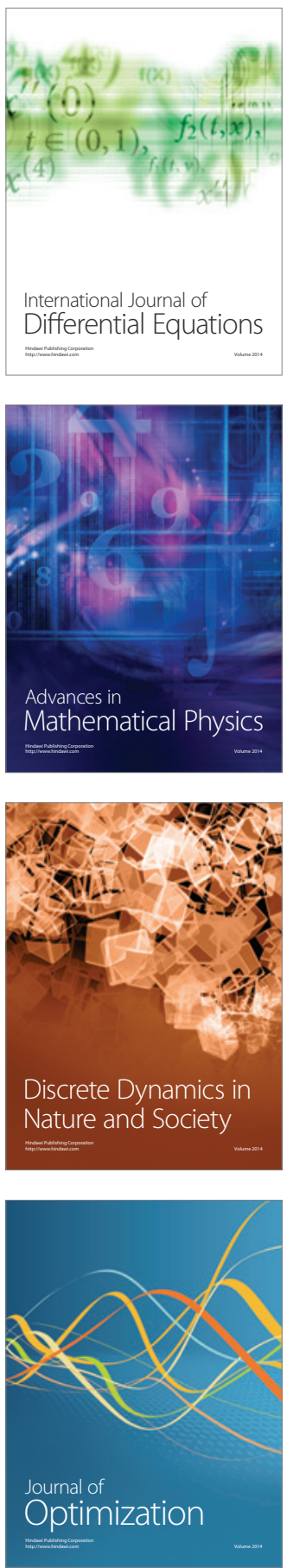